\title{
Static And Dynamic Pupillometric Changes With Silodosin; Selective a1 Adrenoceptor Blocker
}

\section{Umut Karaca ( $\nabla$ drumutkaraca@gmail.com )}

Suleyman Demirel University Faculty of Medicine Department of Ophthalmology Isparta/Turkey

\section{Engin Kaya}

University of Health Sciences, Gulhane Education and Research Hospital, Department of Urology Ankara /Turkey

\section{Onder Ayyildiz}

University of Health Sciences, Gulhane Education and Research Hospital Department of Ophthalmology Ankara /Turkey

\section{Gokhan Ozge}

University of Health Sciences, Gulhane Education and Research Hospital Department of Ophthalmology Ankara /Turkey

\section{Murat Kucukevcilioglu}

University of Health Sciences, Gulhane Education and Research Hospital Department of Ophthalmology Ankara /Turkey

\section{Fatih Mutlu}

University of Health Sciences, Gulhane Education and Research Hospital Department of Ophthalmology Ankara /Turkey

\section{Research Article}

Keywords: benign prostatic hypertrophia, automated pupillometry, silodosin, a1 adrenergic antagonists, intraoperative floppy iris syndrome

Posted Date: January 20th, 2021

DOI: https://doi.org/10.21203/rs.3.rs-146214/v1

License: (c) (i) This work is licensed under a Creative Commons Attribution 4.0 International License. Read Full License

Version of Record: A version of this preprint was published on March 8th, 2021. See the published version at https://doi.org/10.1186/s12886-021-01894-7. 


\section{Abstract}

Backround: Intraoperative floopy iris syndrome is a variant of the small pupil syndrome that has been observed during cataract surgery in some patients currently or previously treated with the a1 adrenergic blockers. It is important for cataract surgeons to predict the probable complications, preoperatively. The aim of our study is to evaluate the static and dynamic pupil characteristics of patients treated with silodosin - a selective a1 adrenergic blocker- for Benign Prostate Hypertrophy (BPH) and to compare these values with healthy subjects using an automatic quantitative pupillometry system.

Methods: A total of $74 \mathrm{BPH}$ patients treated with silodosin for six months (group 1) and 30 healthy subjects (group 2) were enrolled in this prospective multidisciplinary cross-sectional study. Static and dynamic pupillometric measurements were obtained under optimized conditions and the results were compared between the two groups.

Results: Seventy four male patients with a mean age of $63,35 \pm 7,21$ (46-77) years with BPH treated with silodosin and 30 normal male subjects with a mean age of $63,07 \pm 4,73$ (52-71) years were analyzed. There were statistically significant differences between the groups with regard to scotopic pupil diameter $(P D)$, high photopic $P D$, and low photopic $P D(p<0.001$, for each one). Patient group has statistically significant higher values of amplitude and velocity of pupil contraction and lower values of duration of pupil contraction and latency, duration and velocity of pupil dilation.

Conclusion: Static and dynamic pupil characteristics of subjects treated with silodosin for BPH is different from healthy eyes. In addition, our results may have shed light on understanding the risk for IFIS before cataract surgery and thus surgeons can be on the alert and take precautions.

\section{Backround:}

Silodosin is a new subtype selective Adrenoceptor Blocker (AB) approved for Benign Prostatic Hyperplasia (BPH) and Lower Urinary Tract Symptoms (LUTS). (1,2) As the other a-blockers (alfuzosin, doxazosin, tamsulosin and terazosin), silodosin has some adverse effects including asthenia, dizziness, nasal congestion, arterial (orthostatic) hypotension and intraoperative floppy iris syndrome (IFIS).

Pupillary constriction and dilatation is related with responses of iris to parasympathetic and sympathetic impulses, respectively. (4) Pupillary examination by observing and measuring pupil size, shape, symmetry, response to light and response to near reflex can help clinicians to diagnose many ocular and neurological disorders like IFIS. (5) IFIS is a variant of the small pupil syndrome that has been observed during cataract surgery in some patients currently or previously treated with the a1 ABs. (6)

Dynamic pupillometry is an autonomic testing tool for pupillary mesurements. (7) These measurements can be taken in scotopic, mesopic, or photopic conditions statically and dynamically. Recent developments in automated pupillometry devices have enabled quantitative, objective, noninvasive, and repeatable measurements of pupil diameter (PD) as well as the pupillary kinetics. (8) The aim of this 
study was to evaluate the static and dynamic pupil characteristics of patients treated with silodosin for LUTS/BPH and to compare these values with healthy subjects using an automatic quantitative pupillometry system.

\section{Methods:}

Study was designed as a prospective multidisciplinary cross-sectional study and carried out from July 2015 to July 2017 at ophthalmology and urology clinics of a tertiary hospital The study protocol was approved by the Gulhane Education and Research Hospital Clinical Research Ethics Committee and the study was carried out in accordance with the Declaration of Helsinki. Written informed consent was obtained from the each individual participant.

A total of $74 \mathrm{BPH}$ patients treated with silodosin for six months (group 1) and 30 healthy subjects (group 2) were enrolled in the study. BPH patients were examined by Urology Department. The inclusion criteria were as follows; male patients $\geq 45$ years with symptomatic $B P H$, a peak flow rate $(\mathrm{Qmax})$ of $<15 \mathrm{ml} / \mathrm{s}$, International Prostate Symptom Score (IPSS) of $\geq 8$, quality of life score (QLS) of $\geq 3$, and a peak flow rate (Qmax) of < $15 \mathrm{ml} / \mathrm{s}$. The patients with severe hepatic or renal insufficiency, urinary tract infections, urethral stricture, neurogenic bladder, history of urethral or prostatic surgery and history of various alfa blocker were excluded from the study.

All the subjects underwent a comprehensive ophthalmic examination, including the measurement of the uncorrected and best corrected visual acuity, slit-lamp biomicroscopy. Individuals with a history of systemic vasculopathies, ocular inflammation, anisocoria, head/orbital trauma or ocular surgery/laser treatment were excluded. İris and/or pupil abnormalities such as coloboma, anterior - posterior synechia, pseudoexfoliation syndrome; glaucoma, medications that may affect iris mechanics such as tropicamide, cyclopentolate, pilocarpine and narcotic-derived medications; neurological disease or other diseases of the visual pathways; and those who were not cooperative enough to undergo pupillometry examinations were also excluded.

A technician blind to study performed pupillometry measurements using the same automatic quantitative pupillometry system (MonPack One, Vision Monitor System, Metrovision, Pérenchies, France) Average values were selected after three consecutive measurements for each participant using the automaticrelease mode of the device. (Fig. 1) All measurements were performed at the same time period in a day (12:00 am - 01:00 pm) The following parameters were recorded; latency and duration of contraction and dilatation (ms); initial, minimum, maximum and mean pupil diameter (PD) (mm); amplitude of contraction ( $\mathrm{mm}$ ); and contraction and dilatation speed (velocity) of the pupil (mm/s). Static pupillometry measurements were obtained under several illumination levels to measure pupil size in scotopic (0.1 $\mathrm{cd} / \mathrm{m} 2)$, mesopic $(1 \mathrm{~cd} / \mathrm{m} 2)$, low photopic $(10 \mathrm{~cd} / \mathrm{m} 2)$, and high photopic $(100 \mathrm{~cd} / \mathrm{m} 2)$ vision conditions. Scotopic PD, mesopic PD, low photopic PD and high photopic PD values were recorded. In darkness, after five minutes of darkness adaptation, dynamic pupillometry measurements were obtained for a duration of 90 seconds. (Fig. 2) 


\section{Statistical Analysis:}

Statistical Package for Social Sciences (SPSS) version 20.0 for Windows (IBM, New York, USA) was used for data analysis. The statistical significance was set at $p<0.05$. Descriptive statistics were presented as mean \pm standard deviations, frequency distributions and percentages. The normal distribution of the variables was tested using analytical methods (Kolmogorov-Smirnov/ Shapiro-Wilk tests). Independent sample t-test was used to compare quantitative data.

\section{Results:}

Seventy four male patients with a mean age of $63,35 \pm 7,21(46-77)$ years with BPH treated with silodosin and 30 normal male subjects with a mean age of $63,07 \pm 4,73(52-71)$ years were analyzed. There were no significant differences between the groups with regard to age $(p=0.57)$.

Table 1 shows static pupillometry measurements of Group 1 (patient) and Group 2 (control). There were statistically significant differences between the groups with regard to scotopic PD, high photopic PD, and low photopic PD ( $p<0.001$, for each one). Mesopic PD was not statistically significant despite the powerful difference. $(p=0.007)$

Dynamic pupillometric measurements of the groups are shown in Table 2. Patient group has statistically significant higher values of amplitude and velocity of pupil contraction and lower values of duration of pupil contraction and latency, duration and velocity of pupil dilation. On the other hand resting diameter values was not statistically significant but there were a powerful difference between groups. $(p=$ 0.007).There were no significant differences between the groups with regard to latency of pupil contraction $(p=0.895)$

\section{Discussion:}

In this study, we used an automatic system for static and dynamic pupillometry measurements on the cases with BPH treated with silodosin - a selective a-blocker - and healthy subjects to determine the differences in static and dynamic pupil characteristics. To the best of our knowledge, this is the first study to evaluate the static and dynamic pupil characteristics in subjects treated with silodosin using an automatic quantitative pupillometry system (Vision Monitor System, Metrovision).

Pupillary examination including pupil size, shape, symmetry, response to light,and response to near reflex is important before planning intraocular surgery. However, subjective examination of pupillas can be affected by several factors such as illumination and the examiner's experience. (9) Pupillary response to light can be measured by using an automatic pupillometry system with controlling lightening conditions and can be obtained multiple, quantitative measurements. This improves the repeatability of the measurements, solves the problem of examiner-dependent errors and reduces false negative responses. $(10,11)$ 
IFIS is a variant of the small pupil syndrome and was first described by Chang et al. in 2005. (6) Previous studies have shown that tamsulosin and other ABs inhibit phenylephrine-induced mydriasis, causing myosis in almost equal extent and duration with regardless of dose. (12) An important mechanism of IFIS is drug- melanin interaction causing dilator muscle atrophy (13) Silodosin is a novel, more selective alpha-blocker, which is specific to the lower urinary tract and may have fewer side effects than the other alpha-blockers. $(14,15)$ Although this selectivity, Ipekci and Chatterjee reported silodosin-associated IFIS in their cases similar to other AB's. $(16,17)$ Clinically poor dilated and floppy iris during surgery may shrink the visualisation of the surgical field and complicate the surgery. (18) This clinically observed pupillary changes did not ever been observed with quantitative pupillometric analysis before.

The following parameters were measured with automatic pupillometry system: pupil diameter before and after light stimulus; latency, duration, velocity and amplitude of pupillary constriction; velocity, latency and duration of pupillary dilatation. Amplitude and maximum constriction velocity reflect the active parasympathetic part of the light reflex, whereas the dilatation velocity reflects the active sympathetic part (4)

In this study, all static PDs including the scotopic, mesopic low and high photopic PDs were smaller in patient group. Furthermore, the present study found that patients had higher resting PDs than healthy group. Since the pupillary resting diameter reflects the balance between sympathetic and parasympathetic autonomic systems, it can be said as a result of this study that, silodosin disrupts the balance between autonomic systems in the direction of the parasympathetic system. Dogan et al. investigated the effects of tamsulosin hydrochloride and alfuzosin on pupil diameters and reported smaller resting pupil diameter sizes with alfuzosin treatment especially. (19)

This study investigated the pupil dynamics including latency, duration, and velocity of pupil constriction and redilation of patients treated with silodosin. Our results indicated that patients show statistically significantly higher differences with regard to amplitude of pupil contraction, and velocity of pupil contraction. Duration of pupil contraction, and duration, velocity and latency of pupil dilatation values was statistically significantly lower when compared with healthy eyes. Pupillary dynamics (amplitude and velocity of constriction and dilatation) are a function of the balance between sympathetic and parasympathetic tone in which increased sympathetic balance decreases the constriction velocity, whereas increased parasympathetic balance increases it. (20) These results may suggest that silodosin alters the pupillary kinetics and could be responsible from IFIS as a side effect.

This study had a number of limitations. The relatively small number of patients in the control group could affect the validity and importance of the comparisons. The fact that the pupillometry system used in the study requires full compliance of the patients, may affect the results. It is important to have an experienced technician so that this situation does not affect the work. Another disadvantage of the study is that PD differences such as physiological anisocoria can be seen even in completely healthy subjects.

In conclusion, this study revealed that static and dynamic pupil characteristics of subjects treated with silodosin for BPH differs from healthy eyes. .In addition, our results may have shed light on 
understanding the risk for IFIS before cataract surgery and thus surgeons can be on the alert and take precautions. There have been limited studies about comparison of effects of silodosin in the literature so that further longterm studies are required to clarify the effects of silodosin on static and dynamic pupillary functions.

\section{Declarations:}

\section{Ethics approval and consent to participate}

The procedures in this manuscript were conducted ethically in accordance with the tenets of the Declaration of Helsinki. The study protocol was approved by the Gulhane Education and Research Hospital Clinical Research Ethics Committee (29.06.2015/71). Written informed consent was obtained from the each individual participant.

\section{Consent for publication}

All subjects gave consent to publish this manuscript.

\section{Availability of data and materials}

The datasets used and/or analysed during the current study available from the corresponding author on reasonable request. Unfortunately, the data is not publicly available due to local data protection laws.

\section{Competing interests}

All authors declare that they have no conflicts of interest.

\section{Funding}

All authors declare that they did not receive any support in the form of grants, drugs, etc.

\section{Authors' contributions}

Study conceptualization: (FMM); Conduct of study (EK, OA, MK), Data curation (EK, OA), Formal analysis (UK), Writing-original draft (UK, GO), Writing-review \& editing (UK, GO, OA AND FMM)

\section{Acknowledgements}

Soykan Sahin, MD provided statistical consultation to this study

\section{References:}

1. Kawabe K, Yoshida M, Homma Y, Group SCS. Silodosin, a new alpha1A-adrenoceptor-selective antagonist for treating benign prostatic hyperplasia: results of a phase III randomized, placebocontrolled, double-blind study in Japanese men. BJU Int. 2006;98(5):1019-24. 
2. Yoshida M, Homma Y, Kawabe K. Silodosin, a novel selective alpha $1 \mathrm{~A}$-adrenoceptor selective antagonist for the treatment of benign prostatic hyperplasia. Expert Opin Investig Drugs. 2007;16(12):1955-65.

3. Milani S, Djavan B. Lower urinary tract symptoms suggestive of benign prostatic hyperplasia: latest update on alpha-adrenoceptor antagonists. BJU Int. 2005;95 Suppl 4:29-36.

4. Moog P, Eren O, Kossegg S, Valda K, Straube A, Grünke M, et al. Pupillary autonomic dysfunction in patients with ANCA-associated vasculitis. Clin Auton Res. 2017;27(6):385-92.

5. Kawasaki AK. Diagnostic approach to pupillary abnormalities. Continuum (Minneap Minn). 2014;20(4 Neuro-ophthalmology):1008-22.

6. Chang DF, Campbell JR. Intraoperative floppy iris syndrome associated with tamsulosin. J Cataract Refract Surg. 2005;31(4):664-73.

7. Muppidi S, Adams-Huet B, Tajzoy E, Scribner M, Blazek P, Spaeth EB, et al. Dynamic pupillometry as an autonomic testing tool. Clin Auton Res. 2013;23(6):297-303.

8. Tekin K, Sekeroglu MA, Kiziltoprak H, Doguizi S, Inanc M, Yilmazbas P. Static and dynamic pupillometry data of healthy individuals. Clin Exp Optom. 2018;101(5):659-65.

9. Schröder S, Chashchina E, Janunts E, Cayless A, Langenbucher A. Reproducibility and normal values of static pupil diameters. Eur J Ophthalmol. 2018;28(2):150-6.

10. Martucci A, Cesareo M, Napoli D, Sorge RP, Ricci F, Mancino R, et al. Evaluation of pupillary response to light in patients with glaucoma: a study using computerized pupillometry. Int Ophthalmol. 2014;34(6):1241-7.

11. Bootsma S, Tahzib N, Eggink F, de Brabander J, Nuijts R. Comparison of two pupillometers in determining pupil size for refractive surgery. Acta Ophthalmol Scand. 2007;85(3):324-8.

12. Michel MC, Okutsu H, Noguchi Y, Suzuki M, Ohtake A, Yuyama $H$, et al. In vivo studies on the effects of alpha1-adrenoceptor antagonists on pupil diameter and urethral tone in rabbits. Naunyn Schmiedebergs Arch Pharmacol. 2006;372(5):346-53.

13. Goseki T, Ishikawa H, Ogasawara S, Mashimo K, Nemoto N, Taguchi Y, et al. Effects of tamsulosin and silodosin on isolated albino and pigmented rabbit iris dilators: possible mechanism of intraoperative floppy-iris syndrome. J Cataract Refract Surg. 2012;38(9):1643-9.

14. Jung JH, Kim J, MacDonald R, Reddy B, Kim MH, Dahm P. Silodosin for the treatment of lower urinary tract symptoms in men with benign prostatic hyperplasia. Cochrane Database Syst Rev. 2017;11:CD012615.

15. Krivoborodov GG, Tur El. [Efficiency and safety of silodosin for treatment of lower urinary tract symptoms, associated of benign prostatic hyperplasia]. Urologiia. 2019(2):67-72.

16. Chatterjee S, Agrawal D. Silodosin-associated intraoperative floppy iris syndrome. Indian J Ophthalmol. 2017;65(6):538-9.

17. Ipekci T, Akin Y, Hoscan B, Tunckiran A. Intraoperative floppy iris syndrome associated with silodosin. Acta Ophthalmol. 2015;93(4):e306. 
18. Schwinn DA, Afshari NA. alpha(1)-Adrenergic receptor antagonists and the iris: new mechanistic insights into floppy iris syndrome. Surv Ophthalmol. 2006;51(5):501-12.

19. Dogan M, Kutluksaman B, Keles I, Karalar M, Halat AO. The Effects of Systemic Alfuzosin and Tamsulosin Hydrochloride on Choroidal Thickness and Pupil Diameter Sizes in Cases with Benign Prostatic Hyperplasia. Curr Eye Res. 2017;42(12):1638-43.

20. Bremner FD. Pupillometric evaluation of the dynamics of the pupillary response to a brief light stimulus in healthy subjects. Invest Ophthalmol Vis Sci. 2012;53(11):7343-7.

\section{Tables:}

Table-1

Static Pupillometric Results of Two Groups. (PD: Pupil Diameter, $\mathrm{mm}$ : millimeter, ms: milliseconds)

\begin{tabular}{|llll|}
\hline & $\begin{array}{l}\text { Group 1 } \\
\text { (n: 74) }\end{array}$ & $\begin{array}{l}\text { Group 2 } \\
\text { (n:30) }\end{array}$ & P \\
\hline Scotopic PD (mm) & $5,08 \pm 0,28$ & $5,53 \pm 0,86$ & $<0,001$ \\
\hline Mesopic PD (mm) & $4,17 \pm 0,27$ & $4,55 \pm 0,90$ & 0,007 \\
\hline Low Photopic PD (mm) & $3,17 \pm 0,26$ & $3,66 \pm 0,57$ & $<0,001$ \\
\hline High Photopic PD (mm) & $2,18 \pm 0,34$ & $2,85 \pm 0,46$ & $<0,001$ \\
\hline
\end{tabular}

Table-2

Dynamic Pupillometric Results of Two Groups. (mm: millimeter, ms: milliseconds)

\begin{tabular}{|llll|}
\hline & $\begin{array}{l}\text { group 1 } \\
\text { (n:74) }\end{array}$ & $\begin{array}{l}\text { Group 2 } \\
\text { (n:30) }\end{array}$ & p \\
\hline Resting Diameter (mm) & $4,11 \pm 0,22$ & $4,33 \pm 0,57$ & 0,007 \\
\hline Amplitude of Pupil Contraction (mm) & $1,70 \pm 0,32$ & $1,41 \pm 0,43$ & $<0,001$ \\
\hline Latency of Pupil Contraction (ms) & $284,2 \pm 22,1$ & $281,8 \pm 97,6$ & 0,895 \\
\hline Duration of Pupil Contraction (ms) & $532,3 \pm 49,5$ & $677,1 \pm 186,2$ & $<0,001$ \\
\hline Velocity of Pupil Contraction (ms) & $5,03 \pm 0,37$ & $4,34 \pm 1,18$ & 0,002 \\
\hline Latency of Pupil Dilation (ms) & $851,1 \pm 101,5$ & $972,2 \pm 183,0$ & $<0,001$ \\
\hline Duration of Pupil Dilation (ms) & $1603,3 \pm 73,5$ & $2921,9 \pm 112,7$ & $<0,001$ \\
\hline Velocity of Pupil Dilation (ms) & $1,70 \pm 0,09$ & $2,42 \pm 0,95$ & $<0,001$ \\
\hline
\end{tabular}


Figures

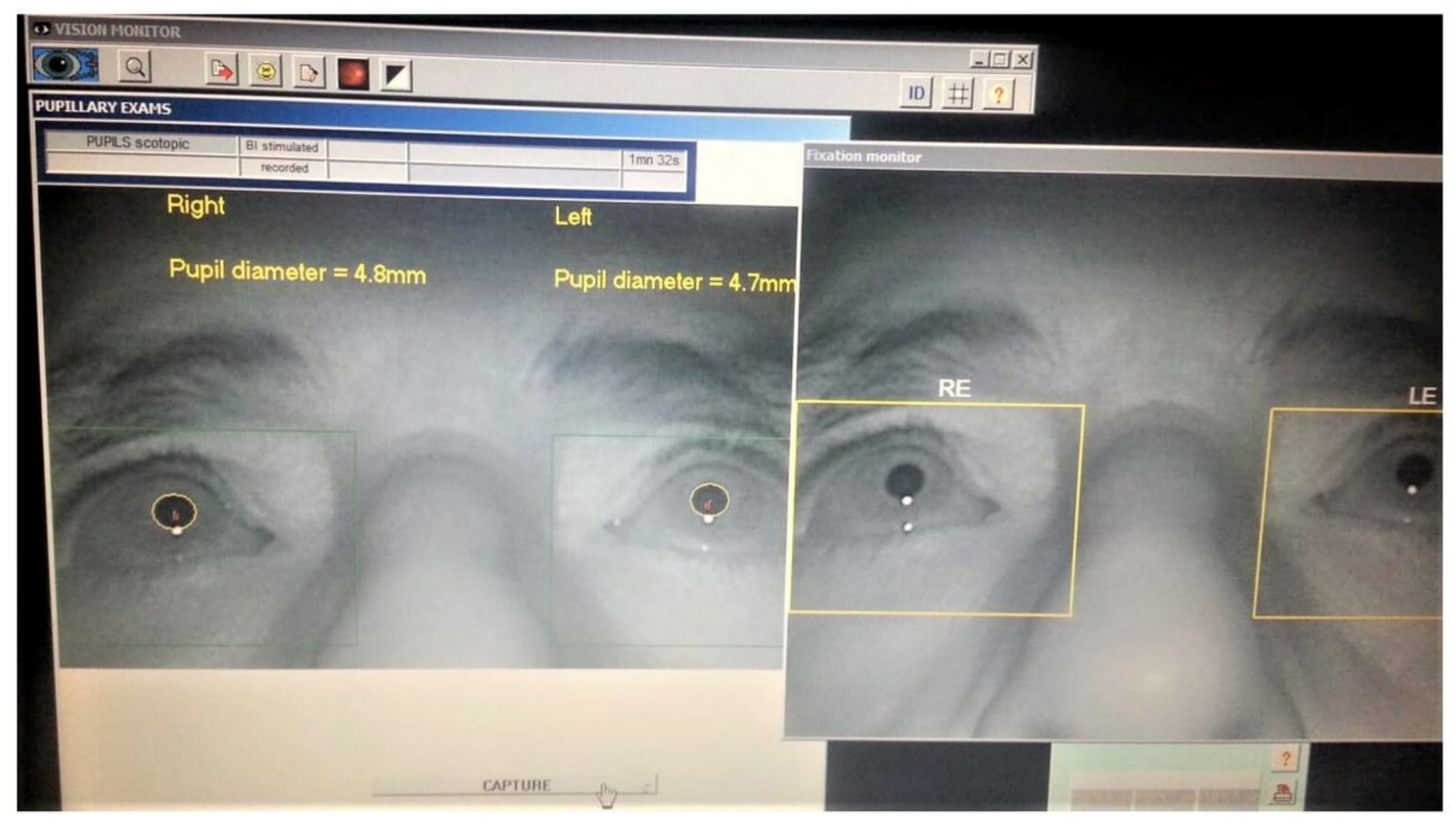

Figure 1

Pupillometry measurement of a patient. 


\section{PUPILLARY EXAMS}

\begin{tabular}{|l|c|l|l|l|}
\hline PUPLS light reflex & Bi stimulated & & & $2 \mathrm{mn} 13 \mathrm{~s}$ \\
\hline & Bi recorded & & & \\
\hline
\end{tabular}

DIAMETER $(\mathrm{mm})$

8

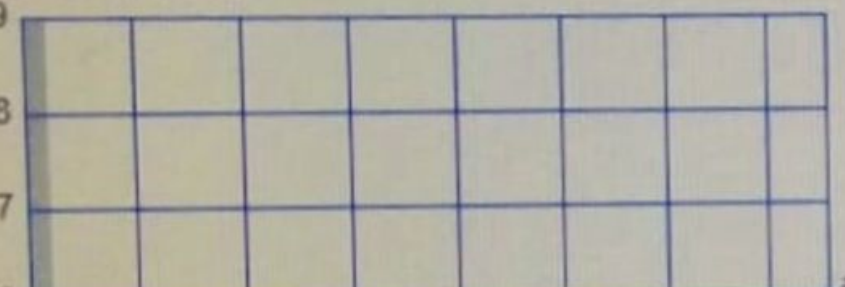

6
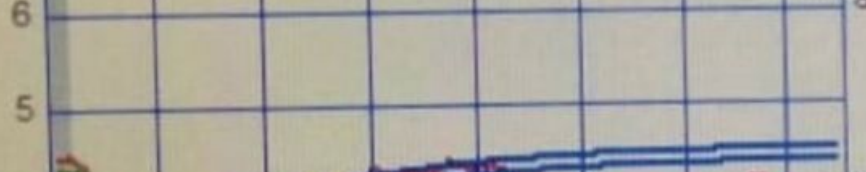

4

4

3

2

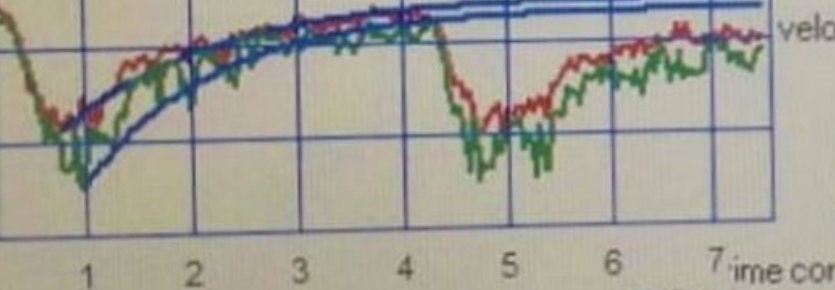

$\begin{array}{lllllll}1 & 2 & 3 & 4 & 5 & 6 & 7 \text { in } \\ & & & & & \text { TIME (s) }\end{array}$

\section{AVERAGED RESPONSE}

OD OS

Valid Responses =

Rejected responses $=$ initial diameter $(\mathrm{mm})=$ amplitude of contraction $(\mathrm{mm})=$ latency of contraction(ms) = duration of contraction(ms) = velocity of contraction $(\mathrm{mm} / \mathrm{s})=$ latency of dilation $(\mathrm{ms})=$ duration of dilation $(\mathrm{ms})=$ velocity of diation $(\mathrm{mm} / \mathrm{s})=$ 2.163 .66 $1.40 \quad 1.19$ result without first response

\section{Figure 2}

Static and Dynamic Pupillometric Results. 